\title{
Models for genetic evaluations of claw health traits in Spanish dairy cattle
}

\author{
M. A. Pérez-Cabal*1 and N. Charfeddine† \\ *Department of Animal Production, Complutense University of Madrid, 28040 Madrid, Spain \\ †CONAFE, Spanish Holstein Association, 28340 Valdemoro, Spain
}

\section{ABSTRACT}

Genetic parameters of 7 claw health traits from Spanish dairy cattle were estimated and the predictive ability of linear and ordinal threshold models were compared and assessed. This study included data on interdigital and digital dermatitis (DE), sole ulcer (SU), white line disease (WL), interdigital hyperplasia (IH), interdigital phlegmon (IP), and chronic laminitis (CL) collected between July 2012 and June 2013 from 834 dairy herds visited by 21 trained trimmers. An overall claw disorder (OCD) was also considered an indicator the absence or the presence of at least 1 of the 6 disorders. Claw health traits were scored as categorical traits with 3 degrees of severity (nonaffected, mild, and severe disorder). Genetic parameters were estimated by fitting both a standard linear model and an ordinal threshold animal model. Around $21 \%$ of cows had at least 1 claw disorder, and the most frequent disorders were SU, DE, WL, and CL. Heritabilities of claw disorders estimated with a linear model ranged from 0.01 (IP) to 0.05 (OCD), whereas estimates from the ordinal threshold models ranged from 0.06 to 0.39 (for IP and $\mathrm{IH}$, respectively). Repeatabilities of claw health estimated with the linear model varied from 0.03 to 0.18 and estimates with the ordinal threshold model ranged from 0.33 to 0.69 . The global trait OCD was correlated with all disorders, except for IH and IP when the linear model was fitted. Two different genetic backgrounds of claw disorders were found. Digital dermatitis showed positive correlations with IH and IP, whereas SU was positively correlated with WL and CL. The predictive ability of the models was assessed using mean squared error and Pearson correlation between the real observation and the corresponding prediction using cross-validation. Regardless of the claw health status, the linear model led to smaller mean squared error. However, differences in predictive ability were found when predicting nonaffected and affected animals. For

Received March 12, 2015.

Accepted July 21, 2015.

${ }^{1}$ Corresponding author: mapcabal@vet.ucm.es most traits, healthy cows were better predicted using the threshold model, whereas the linear model fitted affected cows better. Correlations between the observed data and corresponding predictions support those results ranging from 0.01 to 0.34 . Claw health traits showed enough genetic variance to be included in the selection goal for Spanish Holsteins to select animals with less susceptibility to claw health problems, and we suggest the linear model for implementing genetic evaluations of claw heath traits.

Key words: claw disorder, dairy cow, genetic parameter, linear and ordinal threshold model

\section{INTRODUCTION}

Functionality and health traits were the focus of breeding objectives in the last 3 decades, as described by Miglior et al. (2005). Besides fertility and udder health, locomotion problems are one of the main concerns for dairy farmers worldwide. Claw disorders affect animal welfare but also lead to economic losses due to negative consequences on milk production (Warnick et al., 2001) and fertility of lame cows (Buch et al., 2011). Claw health can be improved by herd management (Pérez-Cabal and Alenda, 2014; Pérez-Cabal and Charfeddine, 2014), but also through genetic selection (Chapinal et al., 2013; Häggman and Juga, 2013; van der Spek et al., 2013).

In Spain, one-third of cows have at least 1 claw disorder, and most of them become chronic over time (Charfeddine and Pérez-Cabal, 2014). Currently, improving claw health in Spanish dairy cattle is being addressed by selecting for feet and legs conformation traits, but, as van der Linde et al. (2010) pointed out, correlations between conformation traits and claw disorders can differ depending on the populations being studied and the conformation traits (Swalve et al., 2008; Chapinal et al., 2013; Häggman and Juga, 2013). Therefore, to include claw health traits in the national breeding goal, the Spanish Holstein Association (CONAFE) implemented a centralized electronic recording system for claw disorders in 2012 called I-SAP (Charfeddine and Pérez-Cabal, 2014). Spanish trimmers either work independently 
or as part of claw trimming companies and service cooperative workers. The Spanish Holstein Association signed an agreement with the most important company in this sector, Anka Hoof Care (Navarra, Spain), to develop a regular electronic recording system of claw health data. At the same time, CONAFE also signed an agreement with Seragro S.C.G. (A Coruña, Spain), an important cooperative service that also started with its own electronic recording system for claw health data in 2012. Information from both sources are preset to be compatible and all data are saved in the same database.

The 2 prerequisites for selection are that the genetic parameters of claw health traits must be known and genetic evaluations must be implemented. The genetic parameters of claw disorders in Holstein cows have already been estimated in some countries, for example by Gernand et al. (2013) in Germany, Häggman and Juga (2013) in Finland, and Stoop et al. (2010) in the Netherlands. Heritabilities have also been estimated in other dairy breeds, such as Norwegian Red (Ødegård et al., 2013) and Ayrshire (Häggman and Juga, 2012). In all cases, despite the low variance associated with these traits, claw health traits were shown to be heritable and can be used in a breeding program provided that a good amount and quality of data are available. Usually, claw data are recorded as binary or categorical traits and, in theory, threshold models are the most suitable to analyze these kind of response variables. However, there are as many authors who used a linear model (Onyiro et al., 2008; Buch et al., 2011; Chapinal et al., 2013) as authors who fitted a threshold model (Koenig et al., 2005; Ødegård et al., 2013; Schöpke et al., 2013). The linear models led to robust estimations despite the violation of assumption of normality when using a categorical response (van der Waaij et al., 2005; Swalve et al., 2008). However, to our knowledge, no literature has compared the goodness of fit of linear and threshold models using cross-validation. Therefore, the objectives of the present study were (1) to estimate genetic parameters of claw disorders in Spanish dairy cattle and (2) to compare the predictive ability of linear and ordinal threshold models using a cross-validation method.

\section{MATERIALS AND METHODS}

\section{Data Collection}

The Spanish Holstein Association provided each trimmer involved in I-SAP a personal tablet with an application called DATPAT for record keeping in farms. The trimmer connects with the database via the internet to download herd animal data and to send information back after each working day. Data recording relies on an easy procedure. For every visit, which can be either scheduled as a maintenance visit or an emergency visit requested by the farmer, the trimmer downloads data from previous visits to the farm and scores disease traits for each cow as follows: 0 for an unaffected cow, 1 for a mild disorder, and 2 for a severe disorder for each claw. Both heifers and lactating cows were scored, although in the current study only data from lactating cows were used. Workshops were held periodically to unify criteria and to train claw trimmers and were open for new trimmers to promote the I-SAP recording system. Mean incidence rates, standard deviations, and graphs showing the evolution of trimmer data over time were discussed in a theoretical session. During a practical session on farms, claw trimmers discussed the recording of claw health data in a set of 15 to 20 cows used as an example. The 6 claw disorders recorded by the claw trimmers were defined as follows.

- Dermatitis (DE): Erosion of the bulb and infection of digital and interdigital skin is recorded as dermatitis in a single trait. Dermatitis is a highly contagious infectious disorder consisting of an ulceration of the skin along the coronary band or on the interdigital ridge of the claw.

- Sole ulcer (SU): An injury through the sole of the claw capsule often complicated by an infection of the corium, with granulation tissue, necrosis, purulent exudates, and separation of the sole horn.

- White line disease (WL): A fissure or a separation, which occurs on the side wall or sole of the claw, enabling foreign material to penetrate and infect the white line region. The corium is affected with a bleeding disorder and, eventually, with necrosis and granulation tissue. Secondary infections with abscess formation are a common sequel.

- Interdigital hyperplasia (IH): An excess epidermal and hypodermal tissue occupying part or all of the interdigital space that usually does not cause lameness unless it becomes extremely large.

- Interdigital phlegmon (IP): A subacute or acute necrotic infection that originates in the interdigital skin leading to cellulitis in the digital region. It presents as severe, acute, or subacute lameness, invariably affecting one limb, with the swelling of the distal soft tissues resulting in the splaying of the toes.

- Chronic laminitis (CL): Results from the acute or subacute laminitis and often appears a few months after the attack of laminitis. Chronic laminitis is a pathophysiologic disturbance in blood flow in the corium that leads to a breakdown of the dermalepidermal junction of the claw. As a consequence, the dorsal wall of the claw can be recognized by a concave shape. 
Table 1. Description of data involved in the study by herds, trimmed and nonaffected cows, number of lactation, and lactation stage

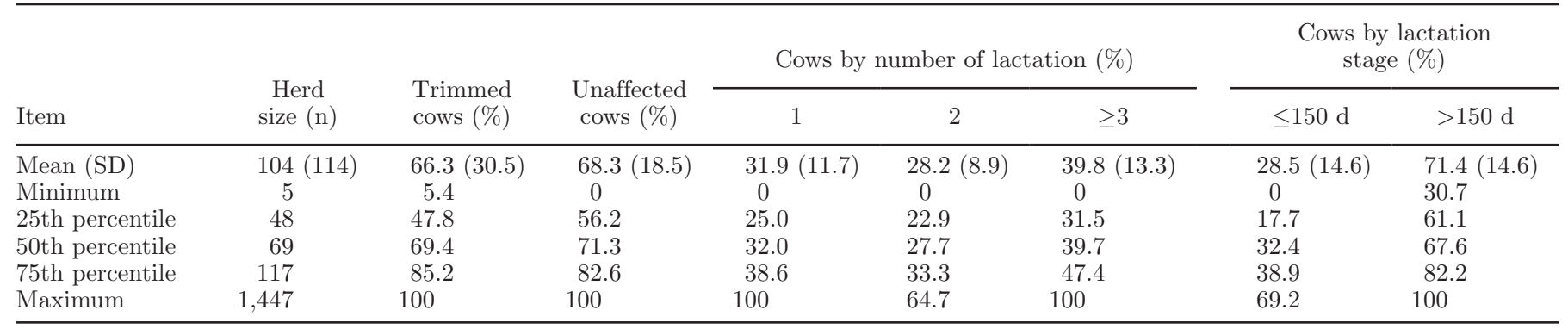

\section{Data Editing}

The 6 claw disorders recorded were analyzed in this study. In addition, an overall claw disorder (OCD) was also considered to indicate the absence or presence of at least 1 of the 6 claw disorders. The OCD was therefore scored from 0 to 2 in such a way that when more than one disorder was noted, the highest score was kept for OCD. In some regions, claw trimming was performed by 2 trimmers at the same time, such that each one trims and scores the front or the hind claws. To define a unique record per animal, and because more than $85 \%$ of the disorders are present in the hind claws, only rear claw disorders were included in our study.

Until August 2013, a total of 132,734 records were gathered by 25 trimmers during 8,523 visits, corresponding to 76,103 cows. To eliminate the training period and to select data registered during a full year, only 78,257 records collected between July 2012 and June 2013 by 21 trimmers in 834 dairy herds during 5,979 visits were initially included in our study. A cow could have more than one event for a specific disorder because trimmers visit the farm more than once a year, with an average of 6.9 visits per herd per year. Two records from the same disorder for the same cow in the same claw within $15 \mathrm{~d}$ between each other were considered as the same disorder and only the first record was used. The first restriction in the editing process was that only data recorded by trimmers with at least 2,000 records throughout the entire period were considered, thus ensuring that trimmers are regularly involved in the recording system $(16,515$ records were removed). Second, claw health data were restricted to herds with at least $50 \%$ of the cows trimmed during the full year to avoid herds with unaffected cows also being trimmed. During preventive visits the cows to be trimmed are usually selected by the farmer to trim all the cows at least once a year. Herds with known high prevalence rates were omitted because these herds have specific management procedures to treat claw health that could bias heritability estimates (van der Spek et al., 2013). We also excluded records from herds with less than 10 records during all the period (6,950 records removed).
Finally, visits with less than 5 cows trimmed per visit were excluded $(4,829$ records removed).

The final data set comprised a total of 49,963 claw health records corresponding to 35,337 Holstein cows, the offspring of 2,759 sires in 566 herds, recorded in 1,679 visits. The final claw disorders records were representative of those from the initial database despite the strict data edits implemented. The average number of animals trimmed per visit was 15 and, on average, each cow had 1.41 records: $47 \%$ had 1 record, $21 \%$ had 2 records, and $3 \%$ had 3 records; the remaining $29 \%$ had up to 15 records. A total of 116,061 animals were included in the pedigree file provided by CONAFE. The average herd size was 104. A description of cows and herds used is shown in Table 1.

\section{Statistical Models}

Genetic parameters were estimated using a linear model and an ordinal threshold model. Many authors studying claw disorders have fitted ordinal threshold models for binary outcomes (Koenig et al., 2005; Ødegård et al., 2013; Oberbauer et al., 2013). However, in our study, claw disorders were classified in 3 categories. The ordinal threshold model is an extension of the binary ordinal threshold model and was previously used in the epidemiological studies of diseases in animal production when the observed variable follows a multinomial distribution (e. g., Vazquez et al., 2009, 2011; Bangera et al., 2014). In the ordinal threshold model it is assumed that the response takes an ordered value $m$ $(1,2, \ldots, m)$ if an underlying continuous variable falls between partially unknown thresholds $\mathrm{T}_{m-1}$ and $\mathrm{T}_{m}$ (Gianola and Foulley, 1983). The probability of getting an observation $\left(y_{i}\right)$ of value $m$ given the systematic and random effects was

$$
\begin{gathered}
\operatorname{Pr}\left(y_{i}=m \mid \text { systematic and random effects }\right)= \\
\Phi\left(T_{m}-\eta_{i}\right)-\Phi\left(T_{m-1}-\eta_{i}\right),
\end{gathered}
$$

where $\Phi($.$) is the standard normal cumulative distribu-$ tion function, $\eta_{i}$ is the linear predictor for an observa- 
tion, and $T_{m}$ and $T_{m-1}$ are thresholds satisfying $-\infty=$ $T_{0}<\ldots<T_{m-1}<T_{m}=\infty$.

The systematic effects included in the models were tested in a study of risk factors in the same population, and Pérez-Cabal and Charfeddine (2014) concluded that number of lactation, age of calving, lactation stage, herd size, and climate conditions were risk factors associated with claw disorders. Cows could have multiple records, and therefore a permanent environmental effect was also considered in the model.

The linear predictor common to the linear model and the ordinal threshold model was

$$
\eta_{i j k l m}=\alpha+\mathrm{HDT}_{i}+\mathrm{LCA}_{j}+\mathrm{LS}_{k}+\mathrm{PE}_{l}+\mathrm{Animal}_{m},
$$

where $\eta_{i j k l m}$ is a function of the expected underlying liability of the claw disorder of a specific cow; $\alpha$ is an intercept; $\mathrm{HDT}_{i}$ is the systematic effect of herd-datetrimmer (1,679 levels); $\mathrm{LCA}_{j}$ is the systematic effect of lactation-calving age (31 levels; up to 3 lactations and up to 13 classes of calving age, from 20 to 85 mo); LS $_{k}$ is the systematic effect of lactation stage (6 levels: from 0 to 60,61 to 120,121 to 180,181 to 240,241 to 305 , and $>305 \mathrm{~d}) ; \mathrm{PE}_{l}$ is the random permanent environmental effect of the $l$ th cow $(35,337$ levels); and Ani$\mathrm{mal}_{m}$ is the random additive genetic effect of the $m$ th animal (116,061 levels). The joint distribution of random effects was $\left(\begin{array}{c}\mathbf{P E} \\ \text { Animal }\end{array}\right) \sim N\left[\mathbf{0},\left(\begin{array}{cc}\mathbf{I} \boldsymbol{\sigma}_{\mathrm{PE}}^{2} & 0 \\ 0 & \mathbf{A} \boldsymbol{\sigma}_{\text {Animal }}^{2}\end{array}\right)\right]$, where $\mathbf{A}$ is the additive genetic relationship matrix between animals; $\mathbf{I}$ is an identity matrix of 35,337 order; and $\boldsymbol{\sigma}_{\text {Animal }}^{2}$ and $\boldsymbol{\sigma}_{\mathrm{PE}}^{2}$ are the corresponding variances.

The final specification for the linear model was

$$
y_{i j k l m}=\eta_{i j k l m}+\varepsilon_{i j k l m},
$$

where $y_{i j k l m}$ is the response of the claw disorder; $\eta_{i j k l m}$ is the function of the expected underlying liability of the claw disorder, and $\varepsilon_{i j k l m}$ is the random error term. The distribution of random residuals was $\sim N\left(\mathbf{0}, \mathbf{I} \sigma_{\varepsilon}^{2}\right)$. The final specification for the ordinal threshold model was

$$
\begin{gathered}
\operatorname{Pr}\left(y_{i j k m}=t \mid \mathrm{HVT}_{i}, \mathrm{LCA}_{j}, \mathrm{DIM}_{k}, \mathrm{PE}_{l}, \text { Animal }_{m}\right)= \\
\Phi\left(T_{t}-\eta_{i j k l m}\right)-\Phi\left(T_{t-1}-\eta_{i j k l m}\right),
\end{gathered}
$$

where $t=0,1,2$ indexes the category of the claw disorder; $\Phi$ is the standard normal cumulative distribution function, and $T_{t}$ and $T_{t-1}$ are fixed thresholds satisfying the order constraint $-\infty<T_{0}<\mathrm{T}_{1}<\infty$. In the ordinal threshold model a random residual effect was assumed to be normally and independently distributed, with mean 0 and variance 1 .

A linear animal model by REML was fitted using the VCE 6.0 software (Neumaier and Groeneveld, 1998; Groeneveld et al., 2010). For the ordinal threshold model, marginal posterior distribution of parameters was estimated using TM software (Legarra et al., 2011) carrying out Makov chain Monte Carlo Gibbs sampling through the chains of 80,000 iterations, discarding the first 30,000 iterations as burn-in period and with a thinning interval of 10 samples. Convergence was examined by the visual inspection of the trace plots using the $\mathrm{R}$ package BOA (Smith, 2007).

Due to computational limitations, the genetic parameters were averaged from the estimations of bivariate analyses where a trait was involved. For all models, heritability $\left(\mathrm{h}^{2}\right)$ and repeatability (rpt) were calculated as $h^{2}=\frac{\sigma_{\text {Animal }}^{2}}{\sigma_{\text {Animal }}^{2}+\sigma_{\mathrm{PE}}^{2}+\sigma_{\varepsilon}^{2}}$ and $\mathrm{rpt}=\frac{\sigma_{\text {Animal }}^{2}+\sigma_{\mathrm{PE}}^{2}}{\sigma_{\text {Animal }}^{2}+\sigma_{\mathrm{PE}}^{2}+\sigma_{\varepsilon}^{2}}$, respectively.

\section{Predictive Ability}

The ability of the models to predict independent observations was assessed using a 10-fold cross-validation (Efron and Tibshirani, 1993). Observations were randomly assigned into 10 subsets of similar size. The model fitted for the predictive ability did not include the permanent environmental effect because not all cows had multiple records, which led to problems with the random assignation into subsets, but repeated observations were retained. As the response variable either for the linear model or the ordinal threshold model was the same, model comparison was approached by computing the Pearson correlation between real observation and the corresponding prediction from the validation data set for all folds together (averages from correlations calculated separately for each fold led to similar values). The mean squared error (MSE) was also used, with the smaller MSE the better model. The MSE was computed as follows:

$$
\mathrm{MSE}=\frac{1}{N} \sum_{f=1}^{10} \sum_{(i j k l) \in f}\left[y_{i j k l}-E\left(y_{i j k l} \mid \hat{\eta}_{i j k l}^{-f}\right)\right]^{2},
$$

where $y_{i j k l}$ is an observation for each of the 7 traits studied; $\hat{\eta}_{i j k l}^{-f}$ is the estimated value of the linear predictor obtained by fitting the corresponding model to a data set that did not include the observations from the fth fold; $(i j k l) \in f$ indicates that the corresponding observation belongs to the $f$ th fold; $E$ is the expectation; and $N$ is the number of observations in the original data set. 
Table 2. Average herd prevalence rates (\%) at herd level, SD, minimum and maximum prevalence rates, and 25th, 50th, and 75th percentile within herds of the 6 claw disorders recorded in Spanish dairy cattle ${ }^{1}$

\begin{tabular}{lrrrrrr}
\hline Item & DE & SU & WL & IH & CL & IP \\
\hline Mean & 8.58 & 16.18 & 7.20 & 0.57 & 3.94 & 0.80 \\
SD & 9.87 & 12.41 & 7.51 & 2.22 & 6.01 & 1.86 \\
Minimum & 0.00 & 0.00 & 0.00 & 0.00 & 0.00 & 0.00 \\
25th percentile & 0.90 & 7.14 & 1.90 & 0.00 & 0.00 & 0.00 \\
50th percentile & 5.55 & 12.98 & 5.22 & 0.00 & 1.65 & 0.00 \\
75th percentile & 12.90 & 22.39 & 10.00 & 0.00 & 5.44 & 0.70 \\
Maximum & 75.00 & 90.00 & 54.50 & 21.80 & 40.40 & 14.30 \\
\hline
\end{tabular}

${ }^{1} \mathrm{DE}=$ digital and interdigital dermatitis; $\mathrm{SU}=$ sole ulcer; $\mathrm{WL}=$ white line disease; $\mathrm{IH}=$ interdigital hyperplasia; CL = chronic laminitis; IP = interdigital phlegmon.

\section{RESULTS AND DISCUSSION}

\section{Prevalence of Claw Health Traits}

The prevalence rates of the 6 claw disorders recorded at herd level are shown in Table 2. The 4 most frequent disorders were $\mathrm{SU}, \mathrm{DE}, \mathrm{WL}$, and $\mathrm{CL}$, whereas IH and IP showed prevalence rates lower than $1 \%$. The prevalence rate at cow level of the 7 traits considered in our study is shown in Table 3. The most frequent disorders, from high to low, were SU, DE, WL, and $\mathrm{CL}$, and around $21 \%$ of the cows had at least 1 claw disorder (OCD). The frequency of OCD varies in the literature depending on the conditions of the study. For example, Laursen et al. (2009) found a low prevalence rate for OCD of $3.52 \%$, which could have been because the study only involved cows in first lactation and the most prevalent disorder in that study was IP. The higher parity number is a risk factor for lameness and for several disorders, such as SU, WL, and CL, whereas cows in first lactation are more prone to DE (Barker et al., 2009; Pérez-Cabal and Alenda, 2014; Pérez-Cabal and Charfeddine, 2014). Conversely, van der Linde et al. (2010) reported that $69 \%$ of cows had at least 1 disorder in $3 \mathrm{yr}$ of study, whereas our data corresponded to only a period of $12 \mathrm{mo}$.

Most of the disorders were classified as mild, whereas severe disorders were very scarce, which suggests a need for a binary response in further studies. As we showed at the herd level, IH and IP had prevalence rates lower than $1 \%$, which explains the rare genetic parameter estimates and results that were obtained and are discussed later in the text. Prevalence of claw disorders varies in the literature, as they depend on herd location, herd facilities, management system, percentage of cows trimmed, and the definition of the traits. Thus, higher frequencies than ours in general were found in the Netherlands (van der Waaij et al., 2005), although in that study the most prevalent disorder was DE (21.7\% digital and $38.7 \%$ interdigital dermatitis). van der Spek et al. (2013) reported $23.8 \%$ of DE (both digital and interdigital) in France, followed by $17.8 \%$ of WLD, and Häggman and Juga (2013) found that WL had a prevalence rate of $10.64 \%$ and was one of the main claw disorders in Finnish Holstein cows. Even the breed can affect the prevalence rates, as Ødegård et al. (2013) reported in Norwegian Red cattle (i.e., 2.9, 2.7, 1.7 , and $0.2 \%$ for $\mathrm{WL}, \mathrm{SU}, \mathrm{DE}$, and IP, respectively).

\section{Heritability and Repeatability of Claw Health Traits}

The heritability estimate for the combined trait OCD was 0.05 for the linear model and 0.11 for the ordinal threshold model. For most claw disorders the estimated heritabilities show that substantial genetic variation is present, which warrants genetic selection to improve claw health. As shown in Table 4, the heritability of claw disorders estimated with the linear model ranged from 0.01 (IP and IH) to 0.05 (OCD). Differ-

Table 3. Prevalence rates at cow level of claw disorders by severity degree in Spanish Holstein cows

\begin{tabular}{lccc}
\hline Item $^{1}$ & $\begin{array}{c}\text { Affected cows } \\
\text { (mild and severe disorders) }\end{array}$ & $\begin{array}{c}\text { Mild } \\
\text { disorder }\end{array}$ & $\begin{array}{c}\text { Severe } \\
\text { disorder }\end{array}$ \\
\hline $\mathrm{DE}$ & 6.55 & 6.54 & 1 \\
$\mathrm{SU}$ & 9.13 & 8.26 & 0.87 \\
$\mathrm{WL}$ & 4.05 & 3.58 & 0.47 \\
$\mathrm{IH}$ & 0.16 & 0.14 & 0.02 \\
$\mathrm{CL}$ & 2.68 & 2.39 & 0.29 \\
$\mathrm{IP}$ & 0.56 & 0.36 & 0.20 \\
OCD & 21.44 & 19.63 & 1.80 \\
\hline
\end{tabular}

${ }^{1} \mathrm{DE}=$ digital and interdigital dermatitis; $\mathrm{SU}=$ sole ulcer; $\mathrm{WL}=$ white line disease $\mathrm{IH}=$ interdigital hyperplasia; $\mathrm{CL}=$ chronic laminitis; IP = interdigital phlegmon; OCD = overall claw disorder. 
ences in the heritability estimates were noted among studies where linear models were fitted. Johansson et al. (2011) reported an estimate of heritability for DE of 0.04 , twice our estimate, and they did not differentiate between digital and interdigital dermatitis as we did. When digital and interdigital dermatitis are treated as different disorders, heritabilities range from 0.01 to 0.09 for digital dermatitis (Onyiro et al., 2008; Stoop et al., 2010; van der Linde et al., 2010) and from 0.0005 to 0.11 for interdigital dermatitis (Swalve et al., 2008; Laursen et al., 2009; van der Linde et al., 2010). Those authors who analyzed both traits simultaneously showed that the heritabilities were very similar for digital and interdigital dermatitis (0.08-0.10). However, the correlation between them varies in the literature; for example, Stoop et al. (2010) reported a high genetic correlation of 0.77 in the first lactation, and Swalve et al. (2008) estimated the genetic correlation at 0.25. For other claw health traits, estimates reported in the literature were similar to our results, ranging from 0.01 to 0.04, such as van der Waaij et al. (2005) for SU, WL, and CL, and van der Spek et al. (2013) for heritability on the observed scale for DE, SU, and WL. Higher estimates than ours were found by Swalve et al. (2008), ranging from 0.05 for WL to 0.14 for CL, and van der Spek et al. (2013), who reported 0.14 for IH.

Estimates of heritability from the ordinal threshold models, expressed in the underlying continuous scale, ranged from 0.06 to 0.39 (for IP and IH, respectively). These values are in accordance with most of the studies on Holstein cattle, such as Swalve et al. (2008), Schöpke et al. (2013), and Gernand et al. (2013), who performed threshold models as well. Oberbauer et al. (2013) reported heritabilities estimated from a threshold model twice as high as those from the literature for digital dermatitis, SU, and WL. They based those differences mainly on the homogeneity of the 3 farms studied and on consistent record keeping. Estimates obtained for different breeds, such as Ødegård et al. (2013) in Nor- wegian Red cattle and Buch et al. (2011) in Swedish Red cows, were also similar, which could mean that the heredity pattern is the same regardless of the breed. As expected, the heritabilities obtained from the threshold model were larger than those estimated using the linear model. The heritabilities, when transformed into an observed scale following Gianola (1979), ranged from 0.00 for IH and IP to 0.05 for SU and OCD (not shown), which are very similar to those that we estimated using the linear model. In our study, both IH and IP showed a prevalence rate less than $1 \%$, making estimation of variance components from an ordinal threshold model difficult, which explains why, for instance, IH presented the highest heritability among all traits when using the threshold model.

Estimated repeatabilities with the linear model ranged from 0.03 (IP) to 0.18 (CL), in accordance with results reported by van der Linde et al. (2010) for Dutch dairy cattle. However, repeatabilities estimated with the ordinal threshold model ranged from 0.33 (IP) to 0.69 (IH; Table 4), which were higher than those estimated from the linear model because of the scale effect, as occurs with heritability. Despite the low values, our results of repeatability for all disorders (except for $\mathrm{IH}$ and IP) suggest that once a cow is diagnosed with any disorder it is more likely to exhibit the same disorder again. It must be noted that in our study around $60 \%$ of the cows had only 2 records. Thus, repeated observations for the same cow within and across lactation are essential to improve accuracy of breeding values.

\section{Genetic Correlations Between Claw Health Traits}

Genetic correlations among 7 traits are shown in Tables 5 and 6, estimated with the linear and the ordinal threshold model, respectively. Genetic correlations with the linear model ranged from -0.69 to 0.98 , whereas with the ordinal threshold model they ranged from -0.64 to 0.97 . Patterns using both models were

Table 4. Heritability, repeatability (rpt), and the corresponding SE (in parentheses) of claw disorders estimated with linear model and corresponding posterior means estimates with the ordinal threshold model

\begin{tabular}{|c|c|c|c|c|}
\hline \multirow{2}{*}{$\begin{array}{l}\text { Claw health } \\
\text { trait }^{1}\end{array}$} & \multicolumn{2}{|c|}{ Linear model } & \multicolumn{2}{|c|}{ Threshold model } \\
\hline & $\mathrm{h}^{2}$ & rpt & $\mathrm{h}^{2}$ & $\mathrm{rpt}$ \\
\hline $\mathrm{DE}$ & $0.02(0.004)$ & $0.10(0.006)$ & $0.14(0.031)$ & $0.42(0.032)$ \\
\hline $\mathrm{SU}$ & $0.04(0.004)$ & $0.17(0.006)$ & $0.15(0.024)$ & $0.51(0.019)$ \\
\hline WL & $0.02(0.003)$ & $0.11(0.005)$ & $0.09(0.021)$ & $0.41(0.030)$ \\
\hline $\mathrm{IH}$ & $0.01(0.002)$ & $0.05(0.004)$ & $0.39(0.068)$ & $0.69(0.155)$ \\
\hline CL & $0.04(0.003)$ & $0.18(0.006)$ & $0.07(0.019)$ & $0.58(0.035)$ \\
\hline IP & $0.01(0.002)$ & $0.03(0.004)$ & $0.06(0.019)$ & $0.33(0.212)$ \\
\hline OCD & $0.05(0.004)$ & $0.15(0.005)$ & $0.11(0.007)$ & $0.38(0.016)$ \\
\hline
\end{tabular}

${ }^{1} \mathrm{DE}=$ digital and interdigital dermatitis; $\mathrm{SU}=$ sole ulcer; $\mathrm{WL}=$ white line disease; $\mathrm{IH}=$ interdigital hyperplasia; $\mathrm{CL}=$ chronic laminitis; $\mathrm{IP}=$ interdigital phlegmon; $\mathrm{OCD}=$ overall claw disorder. 
Table 5. Genetic correlations between claw health traits estimated from a linear model (upper diagonal in bold) and corresponding SE (lower diagonal)

\begin{tabular}{lccccrrr}
\hline Item $^{1}$ & DE & SU & WL & IH & \multicolumn{1}{c}{ CL } & \multicolumn{1}{c}{ IP } & OCD \\
\hline DE & & $-\mathbf{0 . 0 8 3}$ & $\mathbf{- 0 . 3 0 0}$ & $\mathbf{0 . 1 0 1}$ & $-\mathbf{0 . 2 7 0}$ & $\mathbf{0 . 3 3 2}$ & $\mathbf{0 . 3 9 2}$ \\
SU & 0.061 & & $\mathbf{0 . 9 8 0}$ & $\mathbf{0 . 0 0 0}$ & $\mathbf{0 . 9 4 7}$ & $\mathbf{- 0 . 6 8 7}$ & $\mathbf{0 . 9 7 9}$ \\
WL & 0.044 & 0.052 & & $-\mathbf{0 . 2 2 5}$ & $\mathbf{0 . 6 2 7}$ & $\mathbf{- 0 . 0 4 7}$ & $\mathbf{0 . 9 3 0}$ \\
IH & 0.018 & 0.016 & 0.115 & & $-\mathbf{0 . 0 3 4}$ & $\mathbf{- 0 . 6 1 4}$ & $\mathbf{0 . 0 3 5}$ \\
CL & 0.049 & 0.057 & 0.364 & 0.020 & & $\mathbf{0 . 1 7 7}$ & $\mathbf{0 . 7 8 3}$ \\
IP & 0.095 & 0.131 & 0.034 & 0.056 & 0.071 & & $\mathbf{0 . 1 6 0}$ \\
OCD & 0.039 & 0.008 & 0.034 & 0.034 & 0.024 & 0.013 & \\
\hline
\end{tabular}

${ }^{1} \mathrm{DE}=$ digital and interdigital dermatitis; $\mathrm{SU}=$ sole ulcer; $\mathrm{WL}=$ white line disease; $\mathrm{IH}=$ interdigital hyperplasia; $\mathrm{CL}=$ chronic laminitis; IP $=$ interdigital phlegmon; OCD $=$ overall claw disorder.

very similar, although the estimates from the ordinal threshold model were associated with higher standard errors. The global disorder, OCD, was highly correlated with all traits, except with IH and IP when the linear model was fitted. This can be explained because the low frequency of both traits does not fit with the assumption of a normal distribution of the response variable, whereas the ordinal threshold model takes into account the low probability of those events.

The horn-related disorders associated with feeding and metabolic disorders (SU, WL, and CL) showed strong genetic correlations using either the linear model or the threshold model. Dermatitis and IP, considered infectious and associated with hygienic management, were positively correlated and both seemed to be correlated with IH; however, the background of $\mathrm{IH}$ is not clear in the literature. Whereas van der Linde et al. (2010) and Johansson et al. (2011) considered IH as an infectious trait, Schöpke et al. (2013) treated IH as a nonifectious disorder. Moreover, Chapinal et al. (2013) consider IH in a different group from hygienic and metabolic disorders. Regardless of the model used, DE showed negative genetic correlations with WL and CL but close to zero with SU. These results are in accordance with other studies that showed low genetic correlations among traits of groups with a different genetic background; such as Buch et al. (2011), who did not distinguish between digital and interdigital dermatitis, or van der Linde et al. (2010), who reported similar genetic correlations as ours for both types of dermatitis. Contrary to our results, Koenig et al. (2005) found a strong positive genetic correlation between $\mathrm{DE}$ and SU using a linear model, but it must be noted that they only studied digital dermatitis and our trait combines both digital and interdigital dermatitis. As could be expected, sometimes the estimates of correlations where IH and IP are involved showed drastic changes depending on the model used because of the low frequency of those traits in the population studied. Therefore, if we do not take into account the results for IH and IP, our estimates are in accordance with the different genetic background of claw health disorders reported in literature.

\section{Predictive Ability}

The MSE obtained using either linear or ordinal threshold models are shown in Table 7. Regardless of the claw health status, the linear model led to smaller MSE, ranging from 0.01 (IP and IH) to 0.18 (OCD). However, differences in predictive ability were found when predicting the unaffected and affected animals. The threshold model provided less biased predictions for unaffected cows, whereas the linear model tended to predict affected cows much better. As the prevalence rate of claw disorders classified as severe was very low, MSE for severe disorders was 3 times the value obtained for mild disorders, which was very similar to the

Table 6. Posterior means of genetic correlations between claw health traits estimated from an ordinal threshold model (upper diagonal in bold) and corresponding posterior standard errors (lower diagonal)

\begin{tabular}{lccccrrr}
\hline Item $^{1}$ & DE & SU & WL & \multicolumn{1}{c}{ IH } & \multicolumn{1}{c}{ CL } & \multicolumn{1}{c}{ IP } & \multicolumn{1}{c}{ OCD } \\
\hline DE & & $\mathbf{0 . 0 4 9}$ & $\mathbf{- 0 . 3 0 2}$ & $\mathbf{0 . 7 2 1}$ & $\mathbf{- 0 . 1 2 2}$ & $\mathbf{0 . 4 8 3}$ & $\mathbf{0 . 6 1 0}$ \\
SU & 0.137 & & $\mathbf{0 . 7 9 5}$ & $\mathbf{- 0 . 1 0 2}$ & $\mathbf{0 . 7 5 0}$ & $\mathbf{- 0 . 7 3 5}$ & $\mathbf{0 . 9 7 0}$ \\
WL & 0.151 & 0.050 & & $-\mathbf{0 . 4 5 1}$ & $\mathbf{0 . 3 6 3}$ & $\mathbf{- 0 . 6 1 8}$ & $\mathbf{0 . 9 0 7}$ \\
IH & 0.147 & 0.059 & 0.136 & & $-\mathbf{0 . 3 7 4}$ & $\mathbf{- 0 . 6 4 2}$ & $\mathbf{0 . 8 4 9}$ \\
CL & 0.060 & 0.128 & 0.146 & 0.153 & & $\mathbf{0 . 4 4 9}$ & $\mathbf{0 . 3 7 8}$ \\
IP & 0.136 & 0.083 & 0.116 & 0.108 & 0.166 & & $-\mathbf{0 . 2 0 8}$ \\
OCD & 0.008 & 0.008 & 0.020 & 0.032 & 0.161 & 0.195 & \\
\hline
\end{tabular}

${ }^{1} \mathrm{DE}=$ digital and interdigital dermatitis; $\mathrm{SU}=$ sole ulcer; $\mathrm{WL}=$ white line disease; $\mathrm{IH}=$ interdigital hyperplasia; $\mathrm{CL}=$ chronic laminitis; IP = interdigital phlegmon; OCD = overall claw disorder. 
Table 7. Mean squared error for the 7 claw health traits using either the linear model (LM) or the ordinal threshold model (OTM)

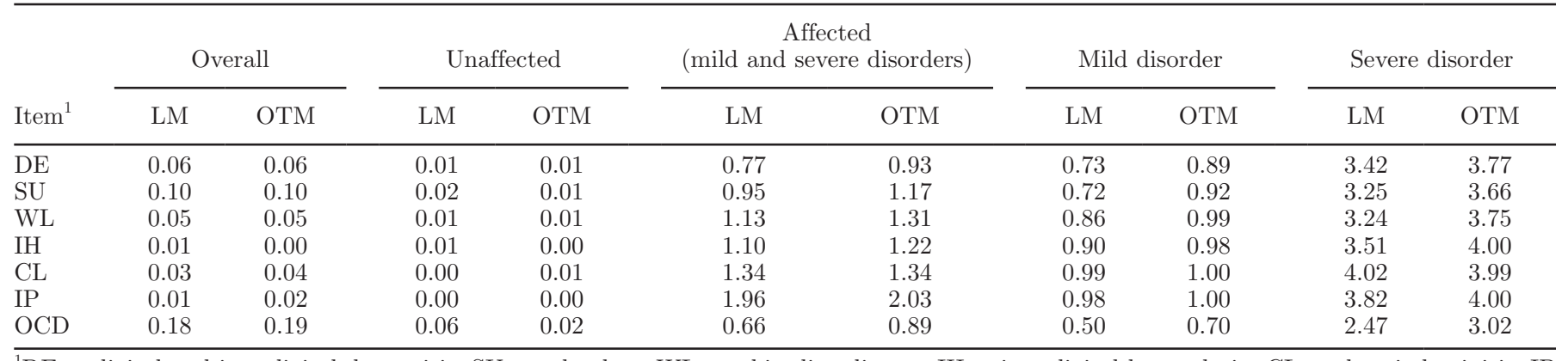

${ }^{1} \mathrm{DE}=$ digital and interdigital dermatitis; $\mathrm{SU}=$ sole ulcer; $\mathrm{WL}=$ white line disease; $\mathrm{IH}=$ interdigital hyperplasia; $\mathrm{CL}=$ chronic laminitis; $\mathrm{IP}$ $=$ interdigital phlegmon; OCD = overall claw disorder.

joint group of affected animals. Therefore, unless the amount of data were large enough and the frequency of severe disorders was high, claw health traits could be scored as a binary response for selection purposes. Correlations between the observed data and corresponding predictions (Table 8) support those results. The Pearson correlations estimated with a linear model ranged from $0.01(\mathrm{IH})$ to $0.34(\mathrm{OCD})$, whereas estimates fitting an ordinal threshold model varied from 0.00 (IH and IP) to 0.33 (OCD). Despite the low correlations, they followed the same trend as the MSE results; that is, for the overall population being studied, the lower MSE and the higher correlations were reached by the linear model. Therefore, the linear model showed higher accuracy for all traits.

The goodness of fit and predictive ability of linear and ordinal threshold models have been studied in animal genetics but, to our knowledge, this is the first time that cross-validation was done for this purpose in the context of claw disorders. Regarding health traits, Vazquez et al. (2011) reported similar results to ours when comparing different models on mastitis traits. As in our study, they found that the ordinal threshold model better predicted the sick cows with multiple mastitis events than the linear model. Vanderick et al. (2014) compared linear and ordinal threshold models to evaluate calving ease in Walloon dairy cattle and reported few differences between both models in terms of MSE, although the correlation between estimated breeding values were slightly better with the linear model, as in our case. Similar results were found by Pérez Enciso et al. (1993) for litter size in Iberian pigs with Poisson versus models. Moreover, Koeck et al. (2012) and Chapinal et al. (2013) fitted linear models based on the results of Neuenschwander (2010), who reported that ordinal threshold models did not improve the goodness of fit compared with linear models. Then, the linear model can be as competitive as the threshold model in claw disorders prediction. In addition, the advantage of linear models in computing requirements (Mrode, 2005) is well known. Therefore, the linear model can be used in the genetic evaluation of claw health traits.

\section{CONCLUSIONS}

Given the heritability estimates we obtained, claw health traits showed enough genetic variance to be included in the breeding goal for Spanish Holsteins to select animals with less susceptibility to claw disorders. In our study, severe disorders were not properly predicted due to their low frequency. Further studies could analyze the effect of mild and severe disorders in economic terms, for instance, but for genetic selection purposes scoring cows as unaffected or affected with claw disorders in further studies would be sufficient to obtain robust predictions given our results for the goodness of fit. The linear model showed better goodness of fit than the ordinal threshold model and it is put forward as the best model to implement a genetic evaluation of claw health traits.

Table 8. Pearson correlation between the real observation and the corresponding prediction from 10-fold crossvalidation using the linear model (LM) and the ordinal threshold model (OTM) for the 7 claw health traits $^{1}$

\begin{tabular}{lccccccc}
\hline Item & $\mathrm{DE}$ & $\mathrm{SU}$ & $\mathrm{WL}$ & $\mathrm{IH}$ & $\mathrm{CL}$ & $\mathrm{IP}$ & OCD \\
\hline LM & 0.23 & 0.21 & 0.16 & 0.01 & 0.02 & 0.03 & 0.34 \\
OTM & 0.16 & 0.15 & 0.06 & 0.00 & 0.01 & 0.00 & 0.33 \\
\hline
\end{tabular}

${ }^{1} \mathrm{DE}=$ digital and interdigital dermatitis; $\mathrm{SU}=$ sole ulcer; $\mathrm{WL}=$ white line disease; $\mathrm{IH}=$ interdigital hyperplasia; $\mathrm{CL}=$ chronic laminitis; $\mathrm{IP}=$ interdigital phlegmon; OCD $=$ overall claw disorder. 


\section{ACKNOWLEDGMENTS}

The authors wish to thank the Spanish dairy association (CONAFE) for their financial support (grant agreement 4155680 UCM-CONAFE), as well as Seragro S.C.G. (A Coruña, Spain), Anka Hoof Care (Navarra, Spain), and all the trimmers, for their vital role in this study. We also thank Lawrence J. C. Baron (freelance proofreader copy-editor based in Madrid, Spain) for his help with the English revision.

\section{REFERENCES}

Bangera, R., J. Odegard, H. Mikkelsen, H. M. Nielsen, M. Seppola V. Puvanendran, H. M. Gjoen, O. J. Hansen, and A. Mortensen. 2014. Genetic analysis of francisellosis field outbreak in Atlantic cod (Gadus morhua L.) using an ordinal threshold model. Aquaculture 420-421(Suppl 1):S50-S56.

Barker, Z. E., J. R. Amory, J. L. Wright, S. A. Mason, R. W. Blowey, and L. E. Green. 2009. Risk factors for increased rates of sole ulcers, white line disease, and digital dermatitis in dairy cattle from twenty-seven farms in England and Wales. J. Dairy Sci. 92:1971-1978.

Buch, L. H., A. C. Sorensen, J. Lassen, P. Berg, J. A. Erikson, J. H. Jakobsen, and M. K. Sorensen. 2011. Hygiene-related and feedrelated hoof diseases show different patterns of genetic correlations to clinical mastitis and female fertility. J. Dairy Sci. 94:1540-1551.

Chapinal, N., A. Koeck, A. Sewalem, D. F. Kelton, S. Mason, G. Cramer, and F. Miglior. 2013. Genetic parameters for hoof lesions and their relationship with feet and leg traits in Canadian Holstein cows. J. Dairy Sci. 96:2596-2604.

Charfeddine, N., and M. A. Pérez-Cabal. 2014. Claw health data recording in Spanish dairy cattle. ICAR, 19-23 May, Berlin, Germany. Accessed Apr. 28, 2015. http://www.icar.org/Documents/ Berlin_2014/index.htm.

Efron, B., and R. J. Tibshirani. 1993. Cross-validation and other estimates of prediction error. Pages 237-255 in An Introduction to the Bootstrap. Chapman \& Hall, New York, NY.

Gernand, E., D. Döhne, and S. König. 2013. Genetic background of claw disorders in the course of lactation and their relationships with type traits. J. Anim. Breed. Genet. 130:435-444.

Gianola, D. 1979. Heritability of polychotomous characters. Genetics 93:1051-1055

Gianola, D., and J. L. Foulley. 1983. Sire evaluation for ordered categorical data with a ordinal threshold model. Genet. Sel. Evol. $15: 201-224$.

Groeneveld, E., M. Kovac, and N. Mielenz. 2010. VCE User's guide and reference manual version 6.0. Accessed Aug. 31, $2015 \mathrm{ftp} / /$ ftp.tzv.fal.de/pub/latest_vce/doc/vce6-manual-3.1-A4.pdf.

Häggman, J., and J. Juga. 2012. The genetic correlation between different claw disorders in Finnish Ayrshire cows. Interbull Bull. $46: 25-27$

Häggman, J., and J. Juga. 2013. Genetic parameters for hoof disorders and feet and leg conformation traits in Finnish Holstein cows. J. Dairy Sci. 96:3319-3325.

Johansson, K., J. K. Eriksson, U. S. Nielsen, J. Pösö, and G. P. Aamand. 2011. Genetic evaluation of claw health in Denmark, Finland and Sweden. Interbull Bull. 43:224-228.

Koeck, A., F. Miglior, D. F. Kelton, and F. S. Schenkel. 2012. Short communication: Genetic association of body condition score with disease resistance in first lactation Canadian Holsteins. Can. J. Anim. Sci. 92:285-289.

Koenig, S., A. Sharifi, H. Wentrot, D. Landmann, M. Eise, and H. Simianer. 2005. Genetic parameters of claw and foot disorders estimated with logistic models. J. Dairy Sci. 88:3316-3325.

Laursen, M. V., D. Boelling, and T. Mark. 2009. Genetic parameters for claw and leg health, foot and leg conformation, and locomotion in Danish Holstein. J. Dairy Sci. 92:1770-1777.
Legarra, A., L. Varona, and E. López de Maturana. 2011. TM Threshold Model. Accessed Aug. 30, 2015. http://snp.toulouse.inra. fr/ alegarra/TMdist.tar.gz.

Miglior, F., B. Muir, and B. Van Doormaal. 2005. Selection indices in Holstein cattle of various countries. J. Dairy Sci. 88:1255-1263.

Mrode, R. A. 2005. Linear Models for the Prediction of Animal Breeding Values. 2nd ed. CAB International, Wallingford, Oxon, UK.

Neuenschwander, T. F. O. 2010. Studies on disease resistance based on producer-recorded data in Canadian Holsteins. $\mathrm{PhD}$ thesis. University of Guelph, Guelph, ON, Canada.

Neumaier, A., and E. Groeneveld. 1998. Restricted maximum likelihood estimation of covariances in sparse linear models. Genet. Sel. Evol. 1:3-26.

Oberbauer, A. M., S. L. Berry, J. M. Belanger, R. M. McGoldrick, J. M. Pinos-Rodriguez, and T. R. Famula. 2013. Determining the heritable component of dairy cattle foot lesions. J. Dairy Sci. 96:605-613.

Ødegård, C., M. Svendsen, and B. Heringstad. 2013. Genetic analyses of claw health in Norwegian Red cows. J. Dairy Sci. 96:7274-7283.

Onyiro, O. M., L. J. Andrews, and S. Brotherstone. 2008. Genetic parameters for digital dermatitis and correlations with locomotion, production, fertility traits, and longevity in Holstein-Friesian dairy cows. J. Dairy Sci. 91:4037-4046.

Pérez-Cabal, M. A., and R. Alenda. 2014. Clinical lameness and risk factors in a Spanish Holstein population. Livest. Sci. 164:168-174.

Pérez-Cabal, M. A., and N. Charfeddine. 2014. Claw lesions and risk factors in Spanish dairy cows. In Proceedings of 10th WCGALP August 17-22, Vancouver, Canada. Poster \#823. Accessed Feb. 27, 2015. https://asas.org/wcgalp-proceedings/species-breedingdairy-cattle- $\% 28$ posters $\% 29$.

Pérez Enciso, M., R. Tempelman, and D. Gianola. 1993. A comparison between linear and Poisson mixed models for litter size in Iberian pigs. Livest. Prod. Sci. 35:303-316.

Schöpke, K., S. Weidling, R. Pijl, and H. H. Swalve. 2013. Relationships between bovine hoof disorders, body condition traits, and test-day yield. J. Dairy Sci. 96:679-689.

Smith, B. J. 2007. boa: An R package for MCMC output convergence assessment and posterior inference. J. Stat. Softw. 21:1-37.

Stoop, W. M., G. de Jong, M. L. van Pelt, and C. van der Linde. 2010. Implementation of a claw health index in the Netherlands. Interbull Bull. 42:95-99.

Swalve, H. H., H. Alkhoder, and R. Pijil. 2008. Estimates for breeding values for sires based on diagnoses recorded at hoof trimming: Relationship with EBV for conformation traits. Interbull Bull. 38:87-90.

van der Linde, C., G. de Jong, E. P. C. Koenen, and H. Eding. 2010. Claw health index for Dutch dairy cattle based on claw trimming and conformation data. J. Dairy Sci. 93:4883-4891.

van der Spek, D., J. A. M. van Arendonk, A. A. A. Vallee, and H. Bovenhuis. 2013. Genetic parameters for claw disorders and the effect of preselecting cows for trimming. J. Dairy Sci. 96:6070-6078.

van der Waaij, E. H., M. Holzhauer, E. Ellen, C. Kamphuis, and G. De Jong. 2005. Genetic parameters for claw disorders in Dutch dairy cattle and correlations with conformation traits. J. Dairy Sci. 88:3672-3678.

Vanderick, S., T. Troch, A. Gillon, G. Glorieux, and N. Gengler. 2014 Genetic parameters for direct and maternal calving ease in Walloon dairy cattle based on linear and ordinal threshold models. J. Anim. Breed. Genet. 131:513-521.

Vazquez, A. I., M. A. Pérez-Cabal, B. Heringstad, M. Rodrigues-Motta, G. J. M. Rosa, D. Gianola, and K. A. Weigel. 2011. Predictive ability of alternative models for genetic analysis of clinical mastitis. J. Anim. Breed. Genet. 129:120-128.

Vazquez, A. I., K. A. Weigel, D. Gianola, D. Bates, M. A. Pérez-Cabal, G. J. M. Rosa, and Y. M. Chang. 2009. Poisson versus ordinal threshold models for genetic analysis of clinical mastitis in US Holsteins. J. Dairy Sci. 92:5239-5247.

Warnick, L. D., D. Jansen, C. L. Guard, and Y. T. Gröhn. 2001. The effect of lameness on milk production in dairy cows. J. Dairy Sci 84:1988-1997. 